\title{
Os préhistorique en Afrique du nord : matière et usages
}

Colette Roubet et Iddir Amara

\section{OpenEdition}

1 Journals

Édition électronique

URL : https://journals.openedition.org/encyclopedieberbere/2837

DOI : 10.4000/encyclopedieberbere.2837

ISSN : 2262-7197

Éditeur

Peeters Publishers

\section{Édition imprimée}

Date de publication : 2 juin 2013

Pagination : 5864-5872

ISBN : 978-2-7584-0194-0

ISSN : 1015-7344

\section{Référence électronique}

Colette Roubet et Iddir Amara, « Os préhistorique en Afrique du nord : matière et usages », Encyclopédie berbère [En ligne], 36 | 2013, document 031, mis en ligne le 12 mars 2021, consulté le 17 février 2022. URL : http://journals.openedition.org/encyclopedieberbere/2837 ; DOI : https://doi.org/ 10.4000/encyclopedieberbere.2837

Ce document a été généré automatiquement le 17 février 2022.

(C) Tous droits réservés 


\title{
Os préhistorique en Afrique du nord : matière et usages
}

\author{
Colette Roubet et Iddir Amara
}

1 La matière osseuse est un support naturel qui a accompagné les hommes préhistoriques. Les Australopithèques lui avaient déjà reconnu avec le lithique diverses aptitudes permettant un usage, étendu aussi à la corne et aux dents (culture ostéodontokeratique créée par R. Dart 1957). En Afrique du Nord, la matière osseuse aurait été exploitée sans interruption depuis le Paléolithique ancien au moins. P. Biberson a fait connaître dans l'Acheuléen moyen de la grotte des Ours (Maroc) deux cas de transformations intentionnelles par «sciage transversal peut être » de côtes d'un mammifère cétacé (famille des Cachalots, détermination C. Arambourg) (Biberson 1957).

2 Les qualités majeures de l'os frais sont la dureté, la robustesse, la compacité et une élasticité intrinsèque. Appréciées et recherchées, elles conviennent à des activités de nutrition, de combustion, de créations par transformation; l'os, matériau utilisable tel quel, facilement débité par percussion, découpé, fendu, incisé, scié, perforé, poli, etc., se présente à l'état frais, cru ou cuit, comme un réservoir nutritif de moelle et de graisse, un bon combustible et une source d'éclairage d'appoint. En Afrique aucun exemple d'utilisation de l'os comme matériau de construction (os d'éléphant par exemple) n'a été signalé, alors qu'en Europe centrale les os de mammouth ont servi à créer des cabanes. Les chasses paléolithiques aux grands mammifères ont procuré de grandes quantités d'ossements; la découpe d'un animal abattu et le partage ont participé à la reconnaissance différentielle (espèce, sexe, âge) des parties anatomiques, musculaires et osseuses, utiles aux projets ultérieurs.

3 Le traitement de l'os à des fins instrumentales, organisé comme le lithique à partir de chaînes opératoires, ne semble pas avoir été synchrone de la pierre durant le Paléolithique inférieur; dans les assemblages on ne décèle pas avant le Paléolithique moyen, pour le moment, de témoins d'une préparation intentionnelle d'outils normés et récurrents ; certes l'éclatement de l'os pour le retrait de la moelle, a toujours fait surgir des esquilles et des éclats aux caractéristiques typiques de chocs, mais ces 
artefacts de percussion ne sauraient fournir à eux seuls les signes d'un traitement intentionnel de la matière, en relation avec des nucléus osseux. Pas plus que certains documents en os fortuitement usés et acuminés par utilisation.

\section{Les travaux pionniers en Afrique du Nord}

4 En Afrique du Nord les recherches techno-typologiques sur l'os débutent vers les années 1960, sans avoir eu connaissance encore des travaux pionniers du chercheur russe S.-A. Semenov (1964) et de son ouvrage Prehistoric technology. Celui-ci traitait déjà des techniques de débitage des matières osseuses en s'appuyant sur la tracéologie. Utilisant aussi un référentiel expérimental, il rapprocha les traces laissées par d'actuelles actions précises sur l'os frais, de celles observées sur les objets préhistoriques, parvenant ainsi à identifier certaines techniques préhistoriques. Le recours à un appareillage grossissant et de grande résolution développera cette voie de recherche.

5 Les travaux généralistes et approfondis conduits par H. Camps-Fabrer (1966) se situent à l'intérieur d'un cadre nord-africain et saharien et un espace chrono-culturel défini : l'Epipaléolithique et le Néolithique. Ils ont le mérite d'inscrire les comportements techniques concernant l'os - et d'autres matières premières - de façon synchrone et parallèle à ceux concernant le lithique. Ainsi ces deux domaines clé participent-ils ensemble à la construction d'une approche globale des productions et comportements observés à une époque donnée. Ne retenir que l'os tronquerait donc la démarche, lui associer la pierre polie, la coquille d'oeuf d'autruche, le derme ossifié* de tortue (Roubet, notice 032), la céramique, la coquille marine élargit la compréhension intégrée des productions à leurs cadres environnementaux et relationnels, renforçant ainsi la pertinence de cette démarche.

\section{Structure de la « liste typologique de l'industrie en os et en ivoire : Epipaléolithique et Néolithique du Maghreb et du Sahara » de H. Camps-Fabrer}

6 Le classement s'appuyant sur une normalisation de la nomenclature occupe une place importante dans l'ouvrage de H. Camps-Fabrer (Ibid. p. 166-170). Ce classement ordonne les documents dans " une liste typologique » sans équivalent alors en France. Chaque type a été présenté avec une définition, des décomptes et des illustrations; cette liste signale sans ambiguïté les marqueurs, ou fossiles directeurs, caractéristiques des principales cultures examinées. La documentation traitée a été presque exhaustive, elle n'avait pas été appréhendée auparavant dans une perspective diachronique et synchronique. Son étude fait ressortir des fondamentaux osseux pertinents et vient confronter ses résultats à ceux établis par J. Tixier (1963) pour le lithique des mêmes ensembles culturels. Les apports de cette forte cohésion conceptuelle restent incontestables dans l'état actuel des connaissances.

7 Cinq grandes familles ont été créées par H. Camps-Fabrer. Elles réunissent les outils tranchants, mousses, perforants, les faucilles et les objets de parure. Dans chaque famille deux lots de documents existent, l'un neutre et récurrent (couteaux plats, lissoirs épais, lissoirs plats, poinçons sauf II et XI, sagaies), l'autre composé des 
marqueurs apparus dans les cultures et faciès de l'Epipaléolithique (Ibéromaurusien et Capsien: Typique, Supérieur) et du Néolithique (Ibid. p. 177-178). Pour nuancer et souligner le potentiel culturel du Capsien Supérieur, celui-ci a été géographiquement scindé en Capsien Supérieur occidental et Capsien Supérieur oriental.

Les outils marqueurs épipaléolithiques :

- de l'ibéromaurusien sont les tranchets à biseau oblique et à biseau concave; ciseaux, épingles à tête globuleuse ;

- du Capsien Typique sont les alènes ;

- du Capsien Supérieur des régions orientales sont les poinçons II et XI, poignards, épingles à tête déjetée, à tête droite, à tête plate et allongée, lamelles osseuses, bâtonnets, corps de faucille (Cadenat 1960), tubes, rondelles et pendeloques ;

- du Capsien Supérieur des régions occidentales sont les aiguilles à canal et les perles tubulaires. L'originalité apparue dans ce faciès installe: "une industrie osseuse « ibéromaurusienne » évoluée » à l'intérieur d'une «industrie lithique dérivée du Capsien 》 (Ibid., p. 173).

Pour le Néolithique, après un découpage en quatre régions géographiques, les régions du Maghreb oriental présentent les marqueurs suivants : couteaux à dos naturels, estèques, lissoirs à extrémité ogivale, spatules, cuillers, aiguilles à chas et labrets. Rien ne distingue le Néolithique des régions telliennes, tandis que pour les régions occidentales, couteaux à dos naturel et cuillers apparaissent. Le Néolithique du Maroc n'est représenté que par deux types : les peignes et les hameçons. Au Sahara deux types sont reconnus : les hameçons courbes et les harpons de tradition soudanaise.

\section{Valorisation des productions osseuses}

10 Sortant du domaine instrumental $\mathrm{H}$. Camps-Fabrer aborde deux autres thèmes insistant sur la valorisation de l'objet en os, rehaussée d'un décor ou obtenue par découpage. Le décor sur os n'a le plus souvent produit qu'une ornementation abstraite, linéaire, sommaire et localisée, soulignée parfois de colorant ocre. Sur quelques outils et objets des incisions groupées ou isolées ont été relevées, certaines de direction parallèle, organisées ou non, continues ou non, hélicoïdales (rares) enfin. Des crans marginaux, des perforations, et des ponctuations couvrent une ou deux faces. Ces incisions concernent une région particulière des objets (poinçons : bases, fût médian et région proximale : couteaux : faces, etc.) du Capsien supérieur et du Néolithique des régions orientales. L'os n'a pas suscité de gravures élaborées; seul le découpage particulier de certaines pièces néolithiques a été signalé. Des contours de figurations anthropomorphes considérées comme des idoles, ont été identifiés au Pic des Singes (Bougie), à Bou Zabaouine (prés d'Ain M'Lila), et à Tanger dans la grotte des Idoles à Achakar. On ne sait à peu près rien des conditions anciennes de découvertes. Ces créations expressives et originales n'ont pas reçu d'analyses technologiques; elles ont été rapprochées de celles découvertes sur le bassin méditerranéen et la tentation d'invoquer une importation de telles pratiques reste une explication comblant provisoirement une méconnaissance des comportements symboliques.

11 Afin de dresser un panorama complet du traitement de l'os, H. Camps-Fabrer a accordé une place spéciale aux ossements humains transformés. On aurait tort de penser que ces supports nous éloignent du domaine de l'os préhistorique : leur étude montre que cette matière les insère dans un ensemble de même nature qui conduisit à traiter 
(découper, perforer, scier) ces parties anatomiques hautement valorisées avec le même référentiel technique. Ainsi, par le biais d'un traitement spécial d'une autre matière osseuse, pour un autre projet exceptionnel, entrepris sur cette substance de l'Etre humain, peut-on s'attendre à découvrir un travail encore plus soigné (nécessitant de recourir à un appareillage grossissant approprié pour l'examiner), des aptitudes techniques plus étendues. Voilà comment se réinstalle le thème technologique au centre de nos préoccupations scientifiques qui vont se développer au $\mathrm{XXI}^{\mathrm{e}}$ siècle. L'usage du crâne humain transformé en masque répond à une coutume universellement attestée, que développèrent durant le Capsien Supérieur en Algérie les Hommes de Mechta-Afalou (escargotière de Mechta-el-Arbi) et les ProtoMéditerranéens (Medjez II, Columnata, et Faïd Souar), et en Libye, ceux de Tripolitaine (cas d'Abiar Miggi). Certains os longs (radius, humérus, cubitus) sont, avec les rondelles crâniennes et une pendeloque, d'autres témoins épipaléolithiques s'inscrivant dans des préoccupations semblables.

Souligner le caractère exhaustif de la documentation instrumentale osseuse étudiée s'imposait; cela restera dans l'histoire de la discipline comme une gageure tenue. Il aurait fallu souligner aussi le rôle déterminant que jouèrent certains sites néolithiques pour rendre compte de la portée des résultats typologiques de H. Camps-Fabrer. Deux grottes ont livré un mobilier exceptionnel par leur état de conservation, la qualité et la diversité de leur collection. Il s'agit d'abord de la grotte de l'Adrar Gueldaman près d'Akbou (de Beaumais et Royer, 1926, BSPF, t. XXIII, p. 223-238). H. Camps-Fabrer (p. 39-40) souligne que ces auteurs figurent parmi les premiers à avoir utilisé une nomenclature précise, empruntée aux gisements européens, et à avoir procédé à des descriptions venant compenser de médiocres résultats photographiques. La reprise des fouilles (2010-2011) dans cette grotte (GLD1) du massif de Gueldaman livre une nouvelle documentation chrono-stratigraphiquement précisée concernant le Néolithique tellien* (Roubet, Kherbouche, N46, EB XXXIV). Comparable à la précédente, elle est d'égal intérêt dans le domaine osseux. Le Damous el-Ahmar dans les Némencha* (Roubet, EB XXIII, N38) est la seconde grotte de référence pour le Néolithique atlasique dont le matériel osseux découvert en 1912 et 1964 servit à structurer la liste typologique de l'industrie osseuse.

\section{Les travaux postérieurs}

13 Le développement de l'expérimentation a redynamisé l'étude des matières osseuses à partir des années 1970. Les apports de documents nombreux, issus de nouvelles fouilles minutieuses, aux contextes chrono-culturels mieux définis, ont permis d'approfondir les recherches.

\section{En Algérie}

Dans le Néolithique pastoral des régions orientales de l'Algérie, la grotte Capéletti* de l'Aurès* a livré une documentation osseuse bien caractérisée et datée se prêtant à une application raisonnée de la liste typologique de H. Camps-Fabrer. Le matériel soumis sans difficulté à cette investigation est apparu plus riche dès lors qu'un rapprochement étroit a été établi avec les documents archéoozologiques. Avant d'entreprendre l'étude faunique, confiée à P.-L. Carter et E. Higgs (1979), C. Roubet (1979) a retiré pièce à pièce 
un ensemble de témoins osseux abandonnés après consommation, ayant tous été déjà intentionnellement transformés par le débitage et la taille. Des expérimentations sur l'os frais ont alors été pratiquées à Alger avec F.-E. Roubet (CRAPE, 1970, 1971). Cette étape clé s'est avérée capitale pour souligner trois séries de faits: (a) le lien étroit unissant la consommation à la transformation ; (b) le rôle du dépotoir-stockage et celui du heu de travail voisin, présents dans cet espace de la grotte ; (c) le lien direct entre les occupants pasteurs de cette grotte et les bêtes domestiques sélectionnées dans leurs troupeaux, pâturant alors dans cet espace montagnard. La grotte Capéletti apparaît à travers cette documentation osseuse aussi comme une aire d'habitat et un heu d'activités intégrées.

Cette collection donne l'occasion d'insister sur le choix très précis des parties anatomiques utilisées et les pratiques du débitage de l'os frais. Ovins, caprins et bovins domestiques ont été abattus au cours des quatre séries d'occupations de la grotte. Leurs ossements furent rejetés dans une poubelle (Roubet, Ibid. p. 162), mais contrairement à d'autres déchets, certains restèrent accessibles et utilisables sur place comme l'attestent de nombreuses pièces de ce cortège documentaire. Aussi ne peut-on pas dire des ossements abandonnés après consommation qu'ils avaient été intentionnellement stockés. Les observations technologiques montrent dans trois familles d'outils (tranchants, mousses et perforants) l'adéquation existant entre un outil façonné et la partie anatomique choisie. Ce sont les os de jeunes ovins adultes plus nombreux que ceux de bovins qui furent sélectionnés. Un corpus d'ébauches taillées a été créé. Pour les outils perforants ce choix systématique s'est ici moins souvent porté sur les fémurs et les humérus, que sur les métapodes. Mis en réserve, ils ont été découpés longitudinalement en deux parties, suivant le sillon carpien de la face dorsale du canon (plutôt qu'en quatre portions), conservant parfois une portion de la poulie articulaire, comme H. Camps-Fabrer l'a souligné. Le sciage longitudinal et la taille par percussion ont été pratiqués. Pour les outils tranchants (couteaux, estèques), les omoplates d'ovins ont aussi été recherchées et découpées de manière à conserver les régions appropriées au futur couteau ou à l'estèque (épine scapulaire rectiligne, pour le dos naturel, et fosse sous-épineuse pour la lame); les côtes épaisses ont été dédoublées par refente, pratiquée dans la zone spongieuse (après sectionnement des extrémités dorsale et ventrale). Deux lames osseuses corticales planes et non courbes ont ainsi été dégagées, la face spongieuse de chacune ayant été soigneusement abrasée, les bords régularisés et polis en biseau. Des expérimentations de ces découpes spéciales ont été pratiquées, à Alger avec le Dr. Ch. Cluzel (CRAPE, 1971).

16 Les éléments d'un diagnostic du débitage sont apportés par plusieurs documents, sciés, rainurés, fendus. Le sciage et le rainurage sont souvent attestés. La percussion lancée a engendré des éclats typiques, dont les empreintes se sont bien conservées sur les ébauches; la retouche marginale régulatrice d'un bord (de l'encoche à la retouche denticulée, voire écailleuse) s'est accompagnée de stigmates et de déchets caractéristiques bien conservés aussi.

La mise en équivalence morphologique d'ébauches taillées préexistantes et de types d'outils polis reconnus dans certaines familles, ne faisait pas de doute dans la démarche analytique de H. Camps-Fabrer, ce corpus d'ébauches taillées était implicitement contenu dans son schéma, mais l'absence de documents ne permit pas d'appuyer ses observations. Les données qu'apportèrent la grotte Capéletti complètent et confortent donc cette démarche sans toutefois se fonder sur une analyse tracéologique, celle des 
traces de cette gestuelle complexe, précise, codée - traces issues de la fabrication et des usages -, par manque d'un équipement approprié disposant d'un fort grossissement et d'une expérimentation approfondie.

Des recherches en relation avec la chaîne opératoire et son potentiel informatif ont été récemment entreprises. Cette approche définit de façon plus dynamique les morphologies comme résultant d'actions techniques ciblées; elle permet d'appréhender les modalités de production et de gestion de l'outillage (Inter alia Campana 1989; Sénépart 1992; Sidéra 1993; David 1999; Liolios 1999; Le Dosseur 2006 ; Legrand 2007). Des choix d'ordre technique, culturel et individuel sont pris en compte, et sont reconnus comme des facteurs déterminants d'un assemblage spécifique pour chaque faciès culturel (Sénépart 1995, p. 211): acquisition et sélection des matières premières, techniques de débitage et de façonnage, morphologies et l'éventuel traitement thermique sont ainsi considérés.

\section{Au Maroc}

19 Au Maroc, une étude techno-typologique a permis de mettre en évidence les principaux caractères des complexes industriels et ceux de l'os en particulier, dans les grottes de Kaf Taht el-Ghar et Dar es-Soltane I, et de la nécropole néolithique de Rouazi à Skhirat* (Kaouane 2002, 2008).

\section{En Tunisie}

20 Sur la côte orientale de Tunisie, l'industrie osseuse capsienne d'un site de plein air de la Sebkhet Halk El-Menjel, désigné par le sigle SHM-1, est la première série à avoir bénéficié de cette approche. L'outillage, peu représenté dans ce site, est composé surtout d'outils pointus obtenus sur des métapodes de ruminants de différente taille, sectionnés en deux ou en quatre et façonnés presque exclusivement par raclage (Mulazzani 2010; Mulazzani et al., à paraître). La récurrence de ces observations portant sur le mobilier osseux d'autres sites capsiens et néolithiques de Tunisie, en cours d'étude, méritait d'être soulignée. On peut envisager provisoirement qu'à côté de spécificités locales, s'est transmise au Néolithique une première caractérisation technique participant à la tradition capsienne du traitement de l'os.

\section{En Libye}

21 On s'étonnera dans cette notice que le matériel osseux de la Libye n'ait pas été étroitement associé à cette démarche ; on retiendra deux séries d'arguments politiques et scientifiques pour expliquer cet état de fait. Au cours du XXe siècle, les recherches en Afrique du Nord occidentale se sont souvent restreintes aux territoires francophones. Dans le domaine de la Préhistoire à l'exception de quelques expéditions internationales, ce sont surtout des équipes britanniques et italiennes qui ont fait connaître les résultats de leurs travaux en Libye et en Egypte. Pour la période concernée par cette notice la documentation découverte et publiée s'est avérée réduite et peu accessible. La grotte d'Haua Fteah reste la monographie de référence (Mc Burney 1967), comme le montrent les études sur le Néolithique de Cyrénaïque (de Faucamberge, EB XXXIV, N43). 


\section{Conclusion}

De nouvelles investigations sur le mobilier osseux nord-africain, livreront certainement un ensemble d'informations précises sur les comportements techniques spécialisés qui révèleront l'étendue des aptitudes et des solutions en réponse à une diversité des matériaux et des besoins.

\section{BIBLIOGRAPHIE}

BIBERSON P., 1957 - « Découverte d'os travaillés dans l'Acheuléen moyen du Maroc atlantique », Actes du $V^{e}$ Congrès de l'Inqua, Madrid-Barcelone.

CADENAT P., 1960 - « Une faucille préhistorique à Columnata », Libyca, VIII, p. 239-252.

CAMPS-FABRER H., 1966 - Matière et art mobilier dans la préhistoire nord-africaine, Paris, AMG

(Mémoires du CRAPE : V, Alger).

CAMPANA D.-V., 1989 - Natufian and Protoneolithic Bone Tools : the Manufacture and Use of Bone

Implements in the Zagros and the Levant, British Archaeological Report International Series 494, Oxford.

DART R.-A., 1957 - The Osteodontokeratic culture of Australopithecus Prometheus, Pretoria, Transvaal Museum.

DAVID E., 1999 - L'industrie en matières dures animales du Mésolithique ancien et moyen en Europe du Nord. Contribution de l'analyse technologique à la définition du Maglemosien, Thèse de doctorat, Université de Paris X.

KAOUANE C., 2002 - Un complément des données sur les cultures néolithiques au Maroc: Etude des outillages osseux des grottes de Kaf Taht el Ghar, de Dar Es Soltan I et de la nécropole de Rouazi à Skhira, Thèse de III ${ }^{e}$ cycle. Institut National des Sciences de l'Archéologie et du Patrimoine, Rabat.

KAOUANE C., 2008 - « Industria osea de Caf Taht el Ghar », in Ramos J., Zouak M., Bernal D., Raissouni B. (Eds.), Las occupaciones humanas de la cueva de Caf Taht el Ghar (Tetuàn). Los productos arqueologicos en el contexto del Estrecho de Gibraltar, Cadiz, p. 134-151.

LE DOSSEUR G., 2006 - La néolithisation au Levant Sud à travers l'exploitation des matières osseuses. Etude techno-économique de onze séries d'industries osseuses du Natoufien au PPNB récent, Thèse de doctorat, Université de Paris I.

LEGRAND A., 2007 - Fabrication et utilisation de l'outillage en matières osseuses du Néolithique de Chypre, British Archaeological Reports, Oxford, Archaeopress.

LIOLIOS D., 1999 - Variabilité et caractéristique du travail des matières osseuses au début de l'Aurignacien: approche technologique et économique, Thèse de doctorat, Université de Paris X. 
MULAZZANI S., 2010 - L'habitat épipaléolithique de SHM-1 et les sites environnants au bord de la sebkhalagune de Halk el Menjel (Hergla - Tunisie) entre le VII et le VI millénaire cal BC, Thèse de doctorat, Université de Paris I.

MULAZZANI S., SIDERA I., (à paraître) - "Technological and typological study of the final Epipalaeolithic assemblage from SHM-1 - Tunisia. A star at characterising the Capsian bone industry", African Archaeological Review.

ROUBET C., 1979 - Economie pastorale préagricole en Algérie orientale. Le Néolithique de tradition capsienne. Exemple l'Aurès, Paris, CNRS (Etudes d'Antiquités Africaines).

SEMENov S.A., 1964 - Prehistoric technology, Londres, Cory, Adam et Mackay, 211 p.

SENEPART I., 1992 - Les industries en matière dure animale de l'Epipaléolithique au Néolithique final dans le Sud-Est de la France, Thèse de Doctorat, Université de Paris X.

SIDERA I., 1993 - Les assemblages osseux en bassin parisien et rhénan du VI au IV millénaire BC. Histoire, techno-économie et culture, Thèse de doctorat, Université de Paris I.

TIXIER J., 1962 - Typologie de l'Epipaléolithique du Maghreb, Paris, AMG (Mémoires du CRAPE : II.)

\section{INDEX}

Mots-clés : Capsien, Corps, Ibéromaurusien, Néolithique, Néolithisation, Outil, Instrument, Paléolithique 